\title{
RANDOMNESS TESTS ON THE SEQUENCE OF ASCAL SEGREGATION CLASSES* IN NEUROSPORA CRASSA
}

\author{
M. R. T. WICKRAMARATNE† and B. C. LAMB \\ Botany Department, Imperial College, London SW7 2BB, England
}

Received 8.vi.76

\begin{abstract}
Summary
Various statistical tests for randomness were made on the order of ascal classes in groups of asci from wild-type $\times$ asco crosses. There was no significant nonrandom clustering of asci of the same segregation class, nor a regular twinning of similar asci. Any apparent observed clustering of similar ascal classes is probably an artefact or due to chance. $2 \times n \chi^{2}$ tests showed that frequencies of individual ascus classes from different perithecia were generally homogeneous, as were second division segregation frequencies. The tests described here for randomness in sequences of occurrences could be of general use in other areas of genetics.
\end{abstract}

\section{INTRODUCTION}

THE unexpected preponderance of certain ascal classes amongst small groups of asci and the occurrence of identical or similar asci consecutively or in small groups separated by one, two or a very few asci of other classes have led many workers to consider the possibility of a non-random clustering or twinning of asci. For example, Mitchell $(1959,1960 a, b)$ suggested that asci developed as closely associated pairs which may be genetically similar although there was no assurance that this pattern of twin asci would be found in all crosses. This twin ascus pair hypothesis seems to contradict the assumption, resulting from the classic cytological studies of Singleton (1953), that each ascus is formed independently, with the consequence that the segregation pattern of each ascus should be independent of that of its neighbours in the ascal cluster. Mitchell (1960b) also obtained indications that the contents of different perithecia from a single cross were sometimes more different than would be expected by chance with respect to relative frequencies of ascal classes. Such heterogeneity could arise if there were a differential maturation or bursting of certain ascal classes (shown in Neurospora by Lamb, 1966, 1967) and if perithecia or groups of asci of different ages were scored. If perithecia were markedly heterogeneous for frequencies of the six ascal classes, it could result in statistically apparent clustering.

The randomness of sequences of ascal segregation classes was investigated using the runs test (Swed and Eisenhart, 1943) to test for clustering of asci of the same segregation class; a method based on Markov chains (Anderson and Goodman, 1957) was used to test for a non-random "twinning" of adjacent asci. The homogeneity between perithecia of relative frequencies of ascal classes was investigated using $2 \times n \chi^{2}$ tests. Wild-type $\times$ asco crosses of Neurospora crassa were used.

* The six segregation classes for one pair of alleles in ordered tetrads.

$\uparrow$ Present address: Genetics Laboratory, Botany School, University of Cambridge, Downing Street, Cambridge CB2 3EA, U.K. 


\section{Materials AND methods}

\section{(i) Experimental}

Crosses of Abbot $4 a \times$ asco (37402) $33 A$ were made using methods described by Lamb (1966). Perithecia were harvested before appreciable ascal dehiscence had occurred, to minimise any differential bursting effects. Asci from many perithecia from a single cross were scored by tallying the segregation class of each of a series of asci in their order of occurrence in an ascal cluster. Ascal classes were numbered I, II, III, IV, V or VI according to Whitehouse and Haldane (1946) and are represented here by Arabic numerals 1-6 respectively. Classes 1 and 2 result from segregation at the first meiotic division and are called MI asci; classes 3-6 result from second division segregation and are called MII asci.

Each continuous series of asci, uninterrupted by incomplete or immature asci, was treated as a separate sample and scores from a single cross were put together. Data were collected from several crosses; in some crosses, results were obtained separately from each perithecium.

\section{(ii) Statistical}

Details of the methods used are given in the Appendices. For further details, see Wickramaratne (1974).

(a) The runs test. The null hypothesis, $\mathrm{H}_{0}$, was that the ascal classes occur in random order, the alternative hypothesis, $\mathrm{H}_{1}$, being that the order of ascal classes deviates from randomness with a predicted direction of deviation (clustering). It was assumed that the samples were statistically independent.

The analysis is based on the order or sequence of events, the number of runs, $u$, in each sample being scored. A run may be defined as a succession of identical symbols which are followed and preceded by different symbols or by no symbols at all. A run may consist of only a single symbol. The total number of runs in a particular sample of any given size gives an indication of the randomness of that sample. For example, if too few runs occur, this would indicate a definite clustering of elements due to lack of independence; too many runs would indicate more mixing than would occur by chance. This procedure does not test whether different runs of the same ascal types are near one another.

The sampling distribution of the values of $u$ which would be expected from repeated random sampling is known. Using this sampling distribution it is possible to decide whether a given sample has more or fewer runs than would probably occur in a random sample. The possibilities can be illustrated as follows for specimen sequences in a hypothetical group of 20 asci containing say 8 of class 1,8 class 2, 2 class 4 and 2 class 5 asci. The end of each " run" of identical symbols is marked by a full stop.

(i) Random: 1.5.1.22.1.2.4.1.5.2.4.11.2222.11.

This has 14 runs and clusters of two or more adjacent identical classes occur occasionally, by chance. Testing for class 2, for example, $m=8, n=12$, $u=9$ (see Appendix for notation). From Swed and Eisenhart (1943), the probability $(P)$ of getting $u$ or less runs by chance is 0.297 . 
(ii) Clustering: 222.11.55.22.1111.44.222.11.

This sequence, with marked clustering, has only 8 runs; two or more adjacent identical classes occur more frequently than expected by chance. For class $2, m=8, n=12, u=6, P=0.025$.

(iii) Twinning: 22.11.55.2222.44.1111.22.11.

This has 8 runs, with the classes occurring in twin pairs which may themselves show random arrangement, mixing or clustering. For class 2, $m=8, n=12, u=6, P=0 \cdot 025$. The runs test does not distinguish twinning from other forms of clustering.

(iv) Mixing: 2.1.5.2.1.2.1.4.2.5.1.2.1.4.2.1.2.1.2.1.

This extreme example has 20 runs; clusters of two or more adjacent identical classes occur less often than by chance. For class $2, m=8$, $n=12, u=16, P=0.999$.

The runs test is for two classes of symbol, not six, so it was applied to each class in turn against all other classes (for example, class 1 asci tested against all non-class 1 asci), and for first division segregation asci ("MI") against second division segregation ("MII") asci. Thus for class 1 asci in the specimen sequences above, there are 11 runs in (i) because sequences such as 2.4 count as a single run, being all " not class 1 "; similarly, in (ii), (iii) and (iv) respectively, there are 6, 6 and 16 runs when testing class 1 .

For each class, information from all the samples was combined using a modification, proposed by Lancaster $(1949,1967)$, of the standard method of combination of probabilities due to Fisher (1936). Details are given in Appendix 1.

(b) The method using Markov chains and maximum likelihood. The null hypothesis, $\mathrm{H}_{0}$, is that the sequence is completely random; the alternative hypothesis, $\mathrm{H}_{1}$, is that there is local non-randomness in the sequence-a one-step dependence with each element depending probabilistically on its predecessor.

The test is based on Markov chains (Anderson and Goodman, 1957). A matrix was constructed by putting a stroke in each cell (see Appendix 2) for the pairwise ascal sequences of that type found in the sample, and then totalling the strokes. An ascus at the beginning or end of a sequence has only one possible partner for consideration as a pair, but non-terminal asci could be part of a pair with the preceding or succeeding ascus: all these possible combinations were scored. Due to the low frequency of second division segregation (MII) classes compared to first division segregation (MI) classes, some of the cells of the matrix were empty or had very small numbers (less than 5) for sample sizes of about 600 . To overcome this effect we used a reduced form of the matrix, combining the MII classes, with $2\left(\mathrm{~L}_{1}-\mathrm{L}_{0}\right)$ being referred to a table of $\chi^{2}$ with 4 degrees of freedom. Large values would provide evidence for rejecting the null hypothesis of randomness.

(c) The $2 \times \mathrm{n}$ homogeneity $\chi^{2}$ test. This was used to test the contents of different perithecia for homogeneity: some samples were analysed only for MI and MII frequencies and others were analysed for the six ordered ascus segregation classes. Because the $\chi^{2}$ test is inaccurate when the expected number in a class is less than 5 , results from all perithecia could not be used in all tests. Nearly all perithecia could be used for testing MI and MII 
frequencies and for classes 1 and 2, but those with less than 20 MII asci were not used for testing classes $3,4,5$ and 6 .

\section{Results}

\section{(i) The runs test}

Table 1 gives the results, for each class, of the combination of probabilities. The probabilities were all greater than 0.05 and therefore within the region of acceptance for the null hypothesis of randomness, so it cannot be rejected in favour of the alternative hypothesis of clustering.

TABLE 1

Runs test : combination of probabilities

Experiment:

Class
1
2
3
4
5
6
MI

Experiment:

Class
1
2
3
4
5
6
MI

(i)

\begin{tabular}{ccc}
\hline$W^{*}$ & $2 k \dagger$ & $P \ddagger$ \\
$233 \cdot 6$ & 204 & $0 \cdot 07$ \\
$214 \cdot 4$ & 208 & $0 \cdot 37$ \\
$208 \cdot 3$ & 228 & $0 \cdot 82$ \\
$240 \cdot 0$ & 262 & $0 \cdot 83$ \\
$241 \cdot 8$ & 256 & $0 \cdot 73$ \\
$234 \cdot 5$ & 230 & $0 \cdot 41$ \\
$168 \cdot 3$ & 158 & $0 \cdot 27$
\end{tabular}

(iii)

\begin{tabular}{rrc}
\multicolumn{1}{c}{ W } & $2 k$ & $P$ \\
$431 \cdot 3$ & 458 & $0 \cdot 81$ \\
$392 \cdot 1$ & 442 & $0 \cdot 96$ \\
$80 \cdot 5$ & 96 & $0 \cdot 75-0 \cdot 90$ \\
$81 \cdot 9$ & 82 & $0 \cdot 25-0 \cdot 50$ \\
$99 \cdot 3$ & 116 & $0 \cdot 87$ \\
123.9 & 128 & $0 \cdot 59$ \\
$291 \cdot 6$ & 302 & $0 \cdot 66$
\end{tabular}

(ii)

\begin{tabular}{rrc}
\hline$W$ & $2 k$ & $P$. \\
$103 \cdot 0$ & 104 & $0 \cdot 51$ \\
$121 \cdot 2$ & 102 & $0 \cdot 09$ \\
$43 \cdot 4$ & 44 & $0 \cdot 30-0 \cdot 50$ \\
$65 \cdot 5$ & 54 & $0 \cdot 10-0 \cdot 20$ \\
$46 \cdot 5$ & 54 & $0 \cdot 70-0 \cdot 80$ \\
$42 \cdot 7$ & 48 & $0 \cdot 50-0 \cdot 70$ \\
$89 \cdot 6$ & 98 & $0 \cdot 70-0 \cdot 80$
\end{tabular}

* $W$ is the sum of $-2 \log _{e} P_{m}$ values, where $P_{m}$ is the median probability computed as $\frac{1}{2}\{P(U<u)+P(U \leqq u)\}$.

$\dagger 2 k$ is the number of degrees of freedom when $k$ probabilities are combined.

$\ddagger P$ is the probability that a $\chi^{2}$ random variable with $2 k$ degrees of freedom is greater than the observed $W$. Clustering would give low $P$ values and "non-random mixing" high ones.

\section{(ii) The method using Markov chains and maximum likelihood}

The results given in table 2 show that all eight samples had $P>0 \cdot 05$. Therefore there was no evidence for rejecting $\mathrm{H}_{0}$, randomness in pairs of adjacent asci, so there are no grounds for searching for assignable causes of clustering in pairs.

Data from the comparable crosses a, b, c, d and e (table 2) were generally homogeneous: for example, for MII frequencies, $\chi_{4}^{2}=3 \cdot 6, P=0 \cdot 3-0 \cdot 5$. The Markov chain data for these particular crosses have therefore been pooled (table 3 ) to get a large enough sample for further tests of randomness within pairs of asci. For the six ordered segregation classes for a pair of 
alleles, one expects a $1: 1$ ratio for classes 1 and 2 , and a $1: 1: 1: 1$ ratio for classes 3, 4, 5 and 6, and good fits to these ratios were usually found in the present data.

Among pairs consisting solely of MI asci, one expects that "twin" arrangements (1.1 and 2.2) and "non-twin" arrangements (1.2 and 2.1)

TABLE 2

Test, using Markov chain method, for non-random " twinning" of adjacent pairs of asci

\begin{tabular}{|c|c|c|c|c|c|}
\hline \multirow[b]{3}{*}{ Data set } & \multirow{3}{*}{$\begin{array}{l}\text { Number of pairs } \\
\text { of asci* }\end{array}$} & \multicolumn{4}{|c|}{ Type of matrix used $\dagger$} \\
\hline & & \multicolumn{2}{|c|}{$6 \times 6, v=25$} & \multicolumn{2}{|c|}{$3 \times 3, v=4$} \\
\hline & & $\chi^{2}$ & $P$ & $\chi^{2}$ & $P$ \\
\hline a & 2005 & $37 \cdot 5$ & $0 \cdot 05-0 \cdot 1$ & $9 \cdot 5$ & $0 \cdot 05-0 \cdot 1$ \\
\hline b & 1900 & $32 \cdot 3$ & $0 \cdot 1 \quad-0 \cdot 2$ & $6 \cdot 3$ & $\begin{array}{lll}0.1 & -0 \cdot 2\end{array}$ \\
\hline c & 701 & - & - & $3 \cdot 0$ & $\begin{array}{lll}0.5 & -0.7\end{array}$ \\
\hline d & 584 & $\ldots$ & - & $3 \cdot 8$ & $\begin{array}{lll}0.3 & -0.5\end{array}$ \\
\hline $\mathrm{e}$ & 675 & - & - & 1.9 & $\begin{array}{lll}0.7 & -0.8\end{array}$ \\
\hline f & 514 & - & - & 1.7 & $0.7-0.8$ \\
\hline g & 356 & _- & - & 1.5 & $0.8-0.9$ \\
\hline h & 917 & - & - & $2 \cdot 0$ & $\begin{array}{lll}0.7 & -0.8\end{array}$ \\
\hline
\end{tabular}

* See Materials and methods (b).

$\uparrow$ With the two large samples, $a$ and $b$, the full $6 \times 6$ matrix could be tested. With the smaller samples, there were too few asci in some classes for valid testing of the $6 \times 6$ matrix, so a reduced form, $3 \times 3$, was used, pooling the second division segregation classes. $\nu$, Number of degrees of freedom.

TABLE 3

Pooled data from Markov chain method

\begin{tabular}{|c|c|c|c|c|c|c|c|c|}
\hline & & & & Ascal & sses* & & & \\
\hline & & 1 & 2 & 3 & 4 & 5 & 6 & Totals \\
\hline & 1 & 1151 & 1103 & 88 & 64 & 86 & 74 & 2566 \\
\hline & 2 & 1049 & 1066 & 98 & 70 & 103 & 101 & 2487 \\
\hline$i$ Ascal & 3 & 85 & 96 & 14 & 9 & 4 & 4 & 212 \\
\hline classes* & 4 & 71 & 75 & 7 & 6 & 6 & 6 & 171 \\
\hline & 5 & 87 & 114 & 5 & 7 & 6 & 4 & 223 \\
\hline & 6 & 84 & 93 & 12 & 2 & 10 & 5 & 206 \\
\hline Totals & & 2527 & 2547 & 224 & 158 & 215 & 194 & 5865 \\
\hline
\end{tabular}

* When considering a sequence of asci as a series of pairs, the first ascus of each pair is called $i$, the second is called $j$; for example, a pair consisting of a class 1 , then a class 4 ascus would be $i 1, j 4$.

will be equally frequent if non-random twinning is absent. The figures of 2217 and 2152 respectively give a good fit to a $1: 1$ ratio $\left(\chi^{2}=1 \cdot 0, P=0 \cdot 3\right.$ $0.5)$. For pairs consisting solely of MII asci, one expects that twin (3.3, 4.4, 5.5 and 6.6$)$ and non-twin $(3.4,3.5,3.6,4.3, \ldots, 6.5)$ arrangements will show a $1: 3$ ratio if non-random twinning is absent. The totals of $31: 76$ give a good fit $\left(\chi^{2}=0 \cdot 9, P=0 \cdot 3-0 \cdot 5\right)$.

One can also test whether pairs of two MI, two MII, or of one MI and one MII asci occur at random. With the observed MI and MII frequencies of 86.33 and 13.67 per cent respectively, the expected numbers of $\mathrm{MI} / \mathrm{MI}$, 
MII/MII and MI/MII pairs, if non-random twinning is absent, are 4371.5, 109.5 and 1384 respectively, compared with observed numbers of 4369,107 and 1389 , which is a good fit $\left(\chi_{2}^{2}=0.08, P=0.95-0.98\right)$.

Tests on the pooled data are subject to slight inaccuracies if there are minor non-homogeneities between the sets of data but even allowing for such reservations, the three tests above on the pooled data clearly showed no evidence of non-random twinning. There are similar reservations about treating the pooled data as a $6 \times 6$ matrix for testing by the maximum likelihood method (as in table 2 for individual samples), or as a $6 \times 6$ contingency table, but if these tests are done they give $\chi_{25}^{2}=36 \cdot 6, P=0 \cdot 05-0 \cdot 1$, and $\chi_{25}^{2}=35 \cdot 5, P=0 \cdot 05-0 \cdot 1$, respectively, again consistent with no significant non-randomness.

(iii) The $2 \times n \chi^{2}$ homogeneity test

In preliminary experiments, results from perithecia were examined just for homogeneity of second division segregation frequencies: the results were

TABle 4

$2 \times \mathrm{n} \chi^{2}$ tests for homogeneity between different perithecia

\begin{tabular}{|c|c|c|c|c|c|c|}
\hline \multirow[b]{2}{*}{ Ascus type } & \multicolumn{3}{|c|}{ Experiment 1} & \multicolumn{3}{|c|}{ Experiment 2} \\
\hline & $\chi^{2}$ & $\nu$ & $P$ & $\chi^{2}$ & $\nu$ & $P$ \\
\hline Class 1 & $16 \cdot 4$ & 15 & $0 \cdot 30-0 \cdot 50$ & $16 \cdot 1$ & 25 & $0.90-0.95$ \\
\hline Class 2 & $13 \cdot 1$ & 15 & $0 \cdot 50-0 \cdot 70$ & $16 \cdot 6$ & 25 & $0 \cdot 80-0 \cdot 90$ \\
\hline Class 3 & $20 \cdot 9$ & $8 *$ & $<0.01$ & 0.5 & $2 *$ & $0 \cdot 70-0 \cdot 80$ \\
\hline Class 4 & $6 \cdot 2$ & 8 & $0 \cdot 50-0 \cdot 70$ & 0.5 & 2 & $0 \cdot 70-0 \cdot 80$ \\
\hline Class 5 & $9 \cdot 2$ & 8 & $0 \cdot 30-0 \cdot 50$ & $0 \cdot 7$ & 2 & $0 \cdot 70-0 \cdot 80$ \\
\hline Class 6 & $7 \cdot 3$ & 8 & $0 \cdot 50-0 \cdot 70$ & $4 \cdot 1$ & 2 & $0 \cdot 10-0 \cdot 20$ \\
\hline MII & $20 \cdot 5$ & 15 & $0 \cdot 10-0 \cdot 20$ & $13 \cdot 5$ & $23 \dagger$ & $0.90-0.95$ \\
\hline
\end{tabular}

$\nu$, Degrees of freedom.

* Some perithecia were omitted for classes 3-6 because they had too few MII asci for valid testing of individual classes.

$\dagger$ Two perithecia omitted as they had too few asci for valid MII frequency testing.

homogeneous even at the 20 per cent level of significance. The more detailed comparisons of all segregation classes made in later experiments are shown in table 4.

There was generally homogeneity between perithecia for frequencies of each of the six segregation classes and of MII asci, although one class 3 result showed significant heterogeneity.

\section{Discussion}

There are certain difficulties in the handling and scoring of asci and in the manipulation of the data. (i) The aggregate of asci within a perithecium cannot be scored intact as it is more like a solid sphere than a ring and hence has to be split into smaller clusters to facilitate scoring: this necessitates the treatment of groups of asci from a single perithecium as separate samples and decreases sample size. (ii) The original arrangement of asci in a cluster is sometimes difficult to determine due to possible changes in position when the 
3-dimensional group of asci is mounted as a flat ring; originally adjacent asci sometimes become separated by several others and remain adjacent only at their attached ends. This would reduce the chance of finding significant associations between ascal classes if there were any but would similarly reduce the chance of observers noticing them visually. (iii) When the ascal clusters examined were in the form of a ring, depending on the point at which scoring is started a pair of asci may be scored as adjacent or not. In such instances scoring was started at a point such that adjacent asci of the same class would not be separated: this preserved a uniformity in the method of scoring although slightly reducing the number of runs compared with starting scoring at random in each ring, thus increasing any apparent clustering.

It is very desirable to use all the information available in a sample but this is not always possible. For example, single isolated asci could not be taken into account in either the runs test or the maximum likelihood method: samples of size 2 and those with elements of one type only were also excluded in the former method. However, there was only a comparatively small number of such samples. When all the elements in a sample were of one type they were usually $\mathrm{MI}$ asci: since the results from testing MI classes were no different from those of the other classes, omitting these samples should not make a significant difference to the final result and conclusion. Since the number of MII asci was small compared to MI asci, some samples contained only a single MII ascus. Consequently, testing MI against MII will give a considerable clue about the outcome of testing each MII class against the rest.

Another disadvantage of using the runs test is that in considering merely the number of runs of each of the six types of asci there is a possibility of missing any clustering effect of runs of the same ascal types being near one another. The main disadvantage, according to Swed and Eisenhart (1943), is that the test lacks power.

In using the maximum likelihood method, the same result should be obtained irrespective of the direction of scoring or the point at which analysis of the sample is begun, but no allowance is made for this. A second disadvantage of this method is that the numbers of each class affect the accuracy of the estimation: this necessitated the reduction of the matrix or else the analysis of very much larger samples.

Appreciable heterogeneity between perithecia for frequencies of individual ascal classes, or for MI and MII asci, was not found in the present experiments. If it occurred, such heterogeneity would affect the Markov chain test for randomness as the overall frequency for any one class will not usually be the applicable frequency for any one perithecium or group of asci. The runs test should be less affected.

Further evidence. The randomness of adjacent ascus segregation classes cannot be proved by statistical methods but evidence against clustering of asci of the same class or twinning of asci is available. On examining the data carefully, the asci of one type do not seem to occur in pairs very often. More often than not they seem to occur singly. This view is strengthened by the exceedingly large number of samples where $m=1, n>m$, most of them also having $u=2 m+\mathrm{l}$, in which case the asci could not possibly have occurred in identical pairs. This very strong evidence against twinning or clustering is completely unaffected by misordering of adjacent asci, unlike 
samples with $m>1$. The large number of samples with $P=1$ where $m>1$ also indicates instances in which asci of the class being considered occur singly. Some perithecia had only one, or an odd number, of certain MII ascal classes, which is further evidence against clustering or twinning. Mitchell (1960b) reported that "twins" sometimes consisted of exactly reversed orders in the two asci but no evidence was found here for regular twinnings of this nature.

Considering small samples, according to Swed and Eisenhart (1943), $P(U \leqq u)<0.05$ is evidence of a tendency toward clustering. Since only a very few samples had $P<0 \cdot 05$, the possibility of clustering seems extremely low.

The twin ascus hypothesis or a non-random clustering of asci of the same segregation class also seems incompatible with classical evidence that ascal segregation patterns are determined by meiotic events. Lindegren (1932, 1933) showed that second division segregation frequency was a measure of the frequency of cross-over between a gene and its centromere and gave an indication of the gene-centromere distance on the linkage map. There is generally reasonable agreement between the gene distances calculated from gene-centromere distances of synnemal genes obtained from second division segregation frequency values for each locus and the same gene distance obtained from two- and three-point crosses. Evidence for this was also given by Lindegren $(1932,1933)$.

Further evidence that ascal segregation patterns are determined by meiotic events comes from several sources such as studies of chromosome aberrations and ascal cytology (e.g. Barry, 1972; Perkins, 1972), and of gene conversion.

For the above reasons and from the present data, a non-random clustering of ascal classes seems very unlikely. The various reports of clustering, from personal impressions rather than statistical tests, could be caused by a "mental artefact" in that the observer tends to notice clusters when they occur-albeit by chance-but fails to take much notice of the many nonclustered regions.

\section{Concluusions}

There was no evidence for a non-random clustering or twinning of asci of the same segregation class; any apparent clustering is probably due to chance or may be an artefact. The contents of different perithecia resulting from a single cross or from replicate crosses were homogeneous with respect to the relative frequencies of MI and MII ascal classes.

\section{Appendix 1. The Runs test}

Let $m=$ number of elements of the type being considered, $n=$ number of elements of the other type: for example, when $m=$ number of class 1 asci, $n=$ number of asci of classes 2 to 6 ; when $m=$ number of MI asci, $n=$ number of MII asci. The $m$ and $n$ events are observed in the sequence in which they occur and the value of $u$, the number of runs, is determined.

Small samples. Samples were considered to be small when $m \leqq n \leqq 20$. $U$ is a random variable and a function of $u$. The probability $P$ of $U \leqq u$ in a random arrangement, for each sample where $m \leqq n \leqq 20$ with a range 
of $m$ from 2 to 20 inclusive, was obtained using the tables of Swed and Eisenhart (1943); they refer to $U$ and $u$ as $u$ and $u^{\prime}$ respectively.

Large samples. Samples were considered large if either $m$ or $n$ was greater than 20 . When $m$ or $n$, but not both, was greater than 20 , the probabilities were calculated using the formula described by Swed and Eisenhart, 1943:

$$
P(U \leqq u)=\frac{1}{C_{m}^{m+n}} \sum_{U=2}^{u} f_{U},
$$

where $f_{U}=2 C_{k-1}^{m-1} . C_{k-1}^{n-1}$, when $U=2 k$, i.e., when $U$ is even, and $f_{U}=C_{k-1}^{m-1} \cdot C_{k-2}^{n-1}+C_{k-2}^{m-1} \cdot C_{k-1}^{n-1}$, when $U=2 k-1$, i.e. $U$ is odd.

When both $m$ and $n$ were greater than 20, samples were analysed using the formula:

$$
x=\frac{u-\left\{\frac{2 m n}{m+n}+1\right\}}{\sqrt{\frac{2 m n(2 m n-m-n)}{(m+n)^{2}(m+n-1)}}}
$$

This method and formula are described by Siegel (1956). $x$ is the standardised deviate: corresponding values for the probability $P$ may be obtained by referring to tables of the normal probability function (cf. table 1, Pearson and Hartley 1966). Two other methods were used, for both large and small samples, in the following cases: where $m=1, u=2, P=\frac{2}{n+1}$ and where $u=2 m+1, P=1$ (see Wickramaratne, 1974, for details).

Combination of probabilities. The combination of a number $(k)$ of independent tests of significance is straightforward when dealing with continuous variables, where the sum of the $-2 \log _{e} P$ values is distributed as $\chi^{2}$ with $2 k$ degrees of freedom (Fisher, 1936). However, if the distribution is discontinuous, the $-2 \log _{e} P$ transformation based on the exact test of significance is biased: the test is therefore not immediately applicable. Such biases were eliminated by using the median probability $P_{m}$ and the median value $\chi^{2}$ as suggested by Lancaster $(1949,1967)$.

If we use the median probabilities as our critical values,

$$
W=-2 \sum_{i=1}^{k} \log _{e} \frac{1}{2}\{P(U<u)+P(U \leqq u)\}
$$

can be referred to tables of $\chi^{2}$ on $2 k$ degrees of freedom. A value of $P \leqq 0.05$ was considered as significant evidence for rejecting the null hypothesis of randomness. When $2 k$ exceęded 100 , i.e. $k>50$, the expression $\sqrt{ } 2 W-\sqrt{4 k-1}$ was referred to the tables of the standardised normal distribution, as recommended by Fisher and Yates (1963) under their $\chi^{2}$ distribution tables.

\section{Appendix 2. The method using Markov chains AND MAXIMUM LIKELIHOOD}

Let $n_{i j}$ (for $i=1,2, \ldots, 6$ and $j=1,2, \ldots, 6$ ) be the number of times that a value $i$ is followed by a value $j$. Then $\left\{n_{i j}\right\}$ is a $6 \times 6$ matrix with $n_{i j}$ 
the element in its $i$ th row and $j$ th column $(i, r=1,2, \ldots, 6)$. Let $n_{. j}$ denote the total in column $j$, and $n_{i}$. the total in row $i$, and $n$.. the grand total of all the $n_{i j} \mathrm{~s}$. Then for example

and

$$
n_{.2}=n_{12}+n_{22}+\ldots+n_{62}
$$

$$
n_{2 .}=n_{21}+n_{22}+\ldots+n_{26}
$$

For the null hypothesis of randomness one estimates $P_{j}$, the probability that the next element will be a $j$, irrespective of what its predecessor was. The maximum likelihood estimate of $P_{j}$ is $\frac{n_{. j}}{n_{. .}}$and the maximised log likelihood is then:

$$
L_{0}=\sum_{j=1}^{6} n_{. j} \log \left(n_{. j}\right)-n_{. .} \log \left(n_{. .}\right)
$$

For the alternative hypothesis the maximum likelihood estimate of $P_{i j}$, the probability that the next element will be a $r$ given that its predecessor is an $i$, is $\frac{n_{i j}}{n_{i}}$ and the maximised loglikelihood is then:

$$
L_{1}=\sum_{i=1}^{6} \sum_{j=1}^{6} n_{i j} \log n_{i j}-\sum_{i=1}^{6} n_{i .} \log n_{i}
$$

This estimates $30(=6 \times 5)$ independent parameters. $2\left(L_{1}-L_{0}\right)$ is referred to tables of $\chi^{2}$ with $30-5=25$ degrees of freedom. Large values of $2\left(L_{1}-L\right)$ are evidence for rejecting the null hypothesis of randomness. This result holds asymptotically for large quantities of data.

Acknowledgments. - We wish to thank Miss Priya Wickramaratne and Dr Rodney Coleman for their most helpful advice on the statistical methods, and Dr H. L. K. Whitehouse for critically reading the manuscript. M. R. T. W. thanks the British Council for an Overseas Student Fee Award during the course of this work.

\section{References}

ANDERSON, T. W., AND GOODMAN, L. A. 1957. Statistical inference about Markov Chains. Ann. Math. Statist., 28, 89-110.

BARRY, E. G. 1972. Meiotic chromosome behaviour of an inverted insertional translocation in Neurospora. Genetics, 71, 53-62.

FISHER, R. A. 1936. Statistical Methods for Research Workers. Sixth Edition. Oliver and Boyd. Fisher, R. A., AND yates, F. 1963. Statistical Tables for Biological, Agricultural and Medical Research. Sixth edition. Oliver and Boyd.

LAMB, B. C. 1966. Polarized segregation in Ascomycetes and the differential bursting of asci. Genet. Res., Camb., 7, 325-334.

LAMB, B. G. 1967. The differential maturation of asci and its relevance to recombination studies of Neurospora, Sordaria and similar Ascomycetes. Genet. Res., Camb., 10, 1-12.

LANCASTER, H. O. 1949. The combination of probabilities arising from data in discrete distributions. Biometrika, 36, 370-382.

LANCASTER, H. O. 1967. Query: The combination of probabilities. Biometrics, 23, 840-842. LINDEGREN, c. c. 1932. The genetics of Neurospora-II. Segregation of the sex factors in asci of $\mathcal{N}$. crassa, $\mathcal{N}$. sitophila and $\mathcal{N}$. tetrasperma. Bull. Torrey bot. Club, 59, 119-138.

LINDEGREN, G. C. 1933. The genetics of Neurospora-III. Pure bred stocks and crossingover in $\mathcal{N}$. crassa. Bull. Torrey bot. Club, 60, 133-154.

MTtGhell, M. B. 1959. Detailed analysis of a Neurospora cross. Genetics, 44, 847-856.

MITCHELL, M. B. 1960a. Ascus formation and recombinant frequencies in Neurospora crassa. Genetics, 45, 507-517. 
MITChell, M. B. 1960b. Evidence for non-random distribution of ascal classes in fruiting bodies of Neurospora crassa. Genetics, 45, 1245-1251.

PEARSON, E. S., AND HARTLEY, H. O. 1966. Biometrika Tables for Statisticians. Third edition. Cambridge University Press.

PERKINS, D. D. 1972. An insertional translocation in Neurospora that generates duplications heterozygous for mating-type. Genetics, 71, 25-51.

sIEGEL, s. 1956. Nonparametric Statistics for the Behavioural Sciences. McGraw-Hill, N.Y.

SINGLETON, J. R. 1953. Chromosome morphology and the chromosome cycle in the ascus of Neurospora crassa. Am. F. Bot., 40, 124-144.

SWED, F. S., AND EISENHART, C. 1943. Tables for testing randomness of groupings in a sequence of alternatives. Ann. Math. Statist., 14, 66-87.

WHITEHOUSE, H. L. K., AND HALDANE, J. B. S. 1946. Symmetrical and asymmetrical reduction in Ascomycetes. 7. Genet., 47, 208-212.

wickramaratne, M. R. T. 1974. Gene conversion in Ascobolus immersus and the control of recombination in Neurospora crassa. Ph.D. Thesis, Imperial College, London. 